\begin{tabular}{|c|c|c|c|c|c|c|}
\hline \multirow{4}{*}{ Impact Factor: } & ISRA (India) & $=3.117$ & SIS (USA) & $=0.912$ & ICV (Poland) & $=6.630$ \\
\hline & ISI (Dubai, UAE & $=0.829$ & РИНЦ (Russia & $=0.156$ & PIF (India) & $=1.940$ \\
\hline & GIF (Australia) & $=0.564$ & ESJI (KZ) & $=8.716$ & IBI (India) & $=4.260$ \\
\hline & JIF & $=1.500$ & SJIF (Morocco & $=5.667$ & OAJI (USA) & $=0.350$ \\
\hline
\end{tabular}

SOI: $\underline{1.1 / T A S} \quad$ DOI: $10.15863 / T A S$
International Scientific Journal
Theoretical \& Applied Science
p-ISSN: 2308-4944 (print) $\quad$ e-ISSN: 2409-0085 (online)
Year: $2019 \quad$ Issue: $05 \quad$ Volume: 73
Published: $30.05 .2019 \quad \underline{\text { http://T-Science.org }}$

SECTION 4. Computer science, computer engineering and automation.
QR - Issue

QR - Article
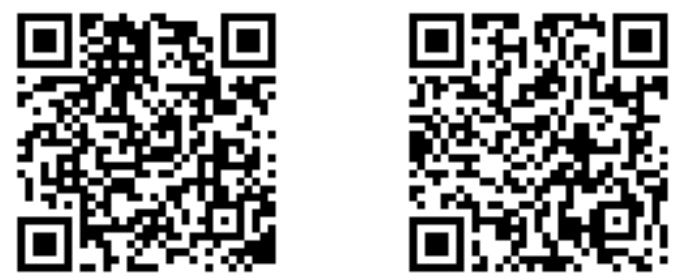

Murat Sauranbayevich Tulenbayev Doctor of Technical Sciences, professor of Computer science, Taraz State University named after M.Kh. Dulaty, Kazakhstan mtulenbaev@mail.ru

Marat Duisenkulovich Manapbaev Master of Engineering science, Software Engineer DLD Taraz State University named after M.Kh. Dulaty, mhclon@mail.ru

Svetlana Temirhanovna Beglerova Candidate of Science, Head of Sector Management of the Educational Process in the

Distance Learning Department (DLD) Taraz State University named after M.Kh. Dulaty, sbeglerova@mail.ru

Adlet Sakenovich Alipbekov Master of Technical Sciences, Software Engineer of the Educational Process in the Distance Learning Department (DLD), Taraz State University named after M.Kh. Dulaty, alipbekov@outlook.com

Anastassiya Andreevna Makovetskaya Bachelor of Technical Sciences, Software Engineer of the Educational Process in the Distance Learning Department (DLD) Taraz State University named after M.Kh. Dulaty, aytsan.m@bk.ru

\title{
COMPARATIVE EVALUATION OF DIGITAL FILTRATION OF SIGNALS IN ANALYTICAL INFORMATION MEASUREMENT SYSTEMS
}

Abstract: The paper are considering the issues of digital filtering of the signals of analytical devices in the composition of information-measuring systems. Two groups of algorithms are compared: a) polynomial filtering in the time domain; $b$ ) wavelet filtering in the spectral region. Boundary estimates and recommendations on the use of algorithms are given.

Key words: analytical devices, chemical-analytical information systems, primary signal processing, polynomial and wavelet filtering of signals, comparative evaluations.

Language: Russian

Citation: Tulenbayev, M. S., Manapbaev, M. D., Beglerova, S. T., Alipbekov, A. S., \& Makovetskaya, A. A. (2019). Comparative evaluation of digital filtration of signals in analytical information measurement systems. ISJ Theoretical \& Applied Science, 05 (73), 473-478.

Soi: http://s-o-i.org/1.1/TAS-05-73-72 Doi: crossef https://dx.doi.org/10.15863/TAS.2019.05.73.72 


\begin{tabular}{|c|c|c|c|c|c|c|}
\hline \multirow{4}{*}{ Impact Factor: } & ISRA (India) & $=3.117$ & SIS (USA) & $=0.912$ & ICV (Poland) & $=6.630$ \\
\hline & ISI (Dubai, UAE & $=0.829$ & РИНЦ (Russia & $=0.156$ & PIF (India) & $=1.940$ \\
\hline & GIF (Australia) & $=0.564$ & ESJI (KZ) & $=8.716$ & IBI (India) & $=4.260$ \\
\hline & JIF & $=1.500$ & SJIF (Morocco & $=5.667$ & OAJI (USA) & $=0.350$ \\
\hline
\end{tabular}

\section{СРАВНИТЕЛЬНЫЕ ОЦЕНКИ ЦИФРОВОЙ ФИЛЬТРАЦИИ СИГНАЛОВ В АНАЛИТИЧЕСКИХ ИНФОРМАЦИОННО-ИЗМЕРИТЕЛЬНЫХ СИСТЕМАХ}

Аннотация: В статье рассматриваются вопросы цифрровой фильтрации сигналов аналитических приборов в составе информационно-измерительных систем. Сравниваются две группь алгоритмов: а) полиномиальная фильтраџия во временной области; б) вейвлет фильтрация в спектральной области. Даются граничнье оченки и рекомендации по использованию алгоритмов.

Ключевые слова: аналитические приборы, химико-аналитические информачионные системы, первичная обработка сигналов, полиномиальная и вейвлет фильтрация сигналов, сравнительные оценки.

\section{Введение.}

Настоящее время характеризуется устойчивой тенденцией к широкому распространению химико-аналитических информационных систем (ХАИС) с универсальными физико-химическими анализаторами состава и свойств вещества, особенно анализаторами спектрального типа (хроматографами, спектрометрами излучения, масс-спектрометрами и др.). Названные аналитические приборы нашли применение для решения задач экологической направленности в составе химико-аналитических комплексов (ХАК) типа ИНЛАН (РФ), Agilent Technologies (USA) и Perkin Elmer. Приборы этих фирм внесены в реестр государственной системы обеспечения единства измерений РК №KZ.02.02.00192-2003.

1.Информацинно-измерительные системы химико-аналитических комплексов.

Основным фактором, затрудняющим использование аналитических приборов (АП), является сложность и разнообразие алгоритмов первичной обработки выходных сигналов. К задачам первичной обработки относятся: дискретное представление сигнала АП, предварительная фильтрация (сглаживание), обнаружение информативного (полезного) участка сигнала, коррекция дрейфа и оценка параметров полезного сигнала [1]. Все алгоритмы первичной обработки можно разбить на две группы:

a) алгоритмы, реализуемые во временной области (без преобразования сигнала);

б) алгоритмы, реализуемые в спектральной области (с преобразованием или отображением сигнала в другое пространство).

Вторая группа по сравнению с первой позволяет достичь более высокой эффективности оценок, что вместе с возможностями современной элементной базы делает эту группу алгоритмов более предпочтительной. Традиционные алгоритмы в большинстве своем относятся к первой группе

Выходной сигнал прибора химикоаналитического комплекса $\mathrm{y}(\mathrm{t})$ в большинстве случаев можно рассматривать как аддитивную смесь полезного сигнала $\mathrm{s}(\mathrm{t}, \mathrm{l})$, помехи $\mathrm{n}(\mathrm{t})$ и базисного сигнала $b(t)[1,2]$ :

$$
y(t)=s(t, l)+n(t)+b(t)
$$

где $\mathbf{l}_{\mathbf{0}}=\left\{1_{0}, 1_{1}, 1_{2}, \ldots, 1_{\mathrm{N}-1}\right\}-$ вектор $\mathrm{N}$ параметров сигнала, подлежащих оценке;

$\mathrm{t}$ - независимая переменная. Параметр $\mathrm{l}_{0}$ выделен из остальных и назван существенным, так как характеризует положение компонента на оси развертки $\mathbf{I}$ и позволяет различить компоненты между собой.

Нахождение по реализациям одного случайного процесса $\boldsymbol{y}(\boldsymbol{t})$, поступающего в обработку, оценок другого, зависимого от него случайного процесса $\boldsymbol{y}^{*}(\boldsymbol{t})$ является задачей фильтрации. Если процессом $\boldsymbol{y}(\boldsymbol{t})$ является сигнал $(1)$, поступающий в обработку, а процессом $y^{*}(t)$ детерминированная (или квазидетерминированная) его часть

$$
y^{*}(t)=s(t)+b(t)
$$

то такая обработка называется сглаживанием сигнала. В процессе сглаживания в той или иной степени устраняется влияние помеховой составляющей $\boldsymbol{n}(\boldsymbol{t})$ сигнала. ХАИС

\section{2. Полиномиальное сглаживание сигналов}

Характеристики шума, прошедшего цифровой фильтр, можно получить, представив входной шум как решетчатый стационарный случайный процесс с корреляционной функцией [3]

$$
\begin{aligned}
& B(l \Delta t)=\frac{1}{q} \sum n(k \Delta t) n((k+l) \Delta t) \\
& B(l \Delta t)^{*}=\sum_{q=-m}^{m} \sum_{r=-m}^{m} h(q \Delta t) h(r \Delta t) \times \\
& \times B[(l+q-r) \Delta t]
\end{aligned}
$$$$
h(q \Delta t)
$$$$
\text { импульсная характеристика }
$$

фильтра. Для нерекурсивного фильтра [3] имеем:

$$
\begin{aligned}
& B(l \Delta t)^{*}=\sum_{q=-2 m}^{2 m} B[(l+q) \Delta t] \times \\
& \times \sum_{k=-m}^{m} h_{k} h_{k-q}
\end{aligned}
$$

Для среднеквадратичного значения шума получим: 


\begin{tabular}{|c|c|c|c|c|c|c|}
\hline \multirow{4}{*}{ Impact Factor: } & ISRA (India) & $=3.117$ & SIS (USA) & $=0.912$ & ICV (Poland) & $=6.630$ \\
\hline & ISI (Dubai, UAE & $=0.829$ & РИНЦ (Russia) & $=0.156$ & PIF (India) & $=1.940$ \\
\hline & GIF (Australia) & $=0.564$ & ESJI (KZ) & $=8.716$ & IBI (India) & $=4.260$ \\
\hline & JIF & $=1.500$ & SJIF (Morocco) & $=5.667$ & OAJI (USA) & $=0.350$ \\
\hline
\end{tabular}

$$
\begin{aligned}
& \sigma_{u}{ }^{*}=\sqrt{B(0)-B(\infty)}= \\
& =\sqrt{\sum_{q=-2 m}^{2 m} B(q \Delta t) \sum_{k=-m}^{m} h_{k}^{2}}
\end{aligned}
$$

При действии на входе фильтра белого шума это выражение упростится:

$$
\sigma_{\phi}^{*}=\sigma_{\phi} \sqrt{\sum_{k=m}^{m} h_{k}^{2}}
$$

Таким образом, ослабление шума пропорционально корню квадратному из числа точек в окне фильтра, при этом даже в случае белого шума на входе фильтра шум на его выходе будет коррелирован.

На рисунке 1 приведены кривые подавления шума полиномиальными фильтрами с $\mathrm{L}=1, \mathrm{~L}=2$ и $\mathrm{L}=4$, где $\mathrm{L}$ - порядок полинома. Наиболее эффективно подавляет шум фильтр скользящего среднего: подавление в этом случае при $\mathrm{m}=2$ такое же, как и фильтром $\mathrm{L}=2$ и $\mathrm{m}=5$ или $\mathrm{L}=4$ и $\mathrm{m}=8$. При больших значениях $\mathrm{m}$ изменение $\mathrm{L}$ и типа фильтра на его эффективность влияет меньше.

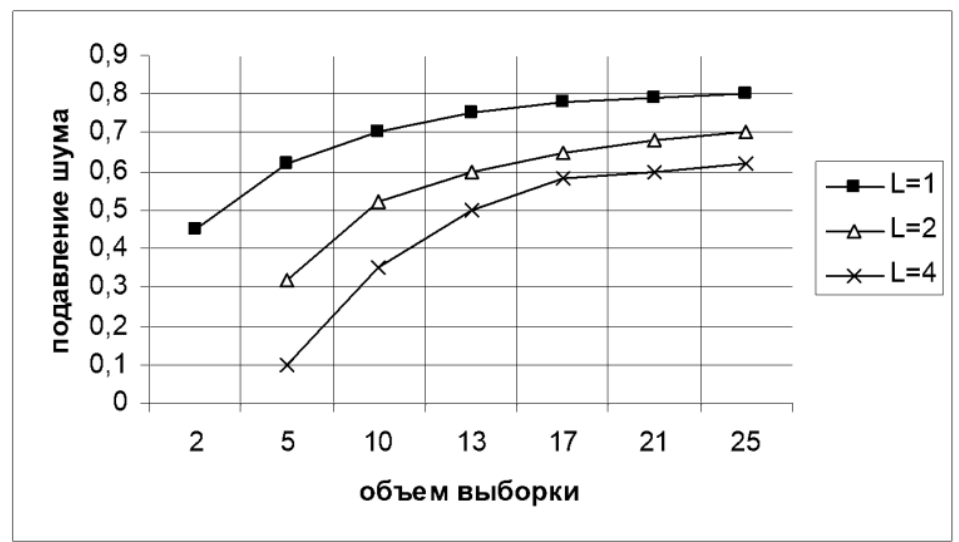

Рисунок 1. Сглаживание нормального шума полиномиальными фильтрами

Так как подавление шумов значительно более эффективно, чем уменьшение амплитуды сигнала вследствие сглаживания, выходной сигнал фильтра должен иметь большее отношение сигнал/помеха. Однако увеличение отношения сигнал/помеха $\xi=q^{*} / q$ относительно невелико. О величине $\xi_{c}$ можно судить по его оценке для оптимального (согласованного) фильтра.

Как показано, например, в [2-4] $q_{\text {iðỗñ }}$ на выходе фильтр достигается, если его переходная (весовая) функция $h(t, \tau)$ является решением интегрального уравнения,

$$
\int B(t, \tau) h(t, \tau) d \tau=f_{i}(t),
$$

где $f(t)$-ожидаемое значение сигнала. Получается, что $h(t, \tau)$ при белом шуме с точностью до множителя совпадает с моделью сигнала, почему фильтр и называется согласованным. При этом $\rho_{\text {макс }}$ (по энергии) равно в случае белого шума:

$$
\rho_{\text {макс }}^{2}=2 E_{c} / G_{0}=E_{c} /\left(\sigma_{\mathrm{m}}^{2} \Delta t\right)
$$

Форма сигнала $s(t)$ на выходе такого фильтра существенно искажается. При обработке аналитической информации согласованная фильтрация используется при обнаружении сигналов и оценивании их параметров методом максимального правдоподобия, поскольку выход такого фильтра $y_{c}(t)$ совпадает с зависящей от сигнала $y(t)$ частью функционала логарифма отношения правдоподобия.

В случае сглаживания Гауссового сигнала в белом шуме, учитывая, что $E_{c}=\sqrt{\pi} A^{2} \mu^{*} \Delta t$ для $\xi_{c}$ из (9) после преобразований получаем:

$$
\xi_{c}=\sqrt{\mu^{*} \sqrt{\pi}}
$$

(где $\mu^{*}=\mu / \Delta t$ ). Таким образом, $\xi$ зависит от $\mu$ (фактически от энергии сигнала). При реальных значениях $\mu^{*}=5 \div 10$ для оценки полиномиальных нерекурсивных фильтров имеем $\xi<\xi_{c}=3 \div 4$ полученными в эксперименте.

При этом следует учитывать, что полиномиальные фильтры эффективны (близки к согласованному), если сигнал в окне фильтра хорошо описывается первыми членами разложения Тейлора. 


\begin{tabular}{|c|c|c|c|c|c|c|}
\hline \multirow{4}{*}{ Impact Factor: } & ISRA (India) & $=3.117$ & SIS (USA) & $=0.912$ & ICV (Poland) & $=6.630$ \\
\hline & ISI (Dubai, UAE & $=0.829$ & РИНЦ (Russia & $=0.156$ & PIF (India) & $=1.940$ \\
\hline & GIF (Australia) & $=0.564$ & ESJI (KZ) & $=8.716$ & IBI (India) & $=4.260$ \\
\hline & JIF & $=1.500$ & SJIF (Morocce & $=5.667$ & OAJI (USA) & $=0.350$ \\
\hline
\end{tabular}

3. Вейвлет фильтрация сигналов химикоаналитических комплексов.

Вейвлет-анализ является разновидностью спектрального анализа[5-9], в котором роль простых колебаний играют вейвлеты. Понятие частоты классического спектрального анализа здесь заменено масштабом, и, чтобы перекрыть "короткими волнами" всю временную ось, введен сдвиг функций во времени. Таким образом, базис вейвлетов - это функции типа $\psi\left(\frac{t-b}{a}\right)$, где: $b-$ сдвиг, $a$ - масштаб. Кроме того, чтобы быть вейвлетом, функция $\psi(t)$ должна иметь нулевую площадь и, еще лучше, равные нулю первый, второй и т.д. моменты. Дискретное вейвлет преобразование (ДВП) основано на теории кратно - масштабного анализа (КМА) сигналов. Положив параметр сдвига $b_{0} \neq 1$ (в классической теории КМА $b_{0}$ всегда равен единице), это понадобится в дальнейшем при изложении теории вейвлетов, применим понятный инженерам спектральный подход к анализу сигналов. В вейвлет-анализе предусматривается применение масштабирующей $\varphi(t)$ и вейвлетной $\psi(t)$ функций, сдвиги и масштабирование которых образуют базисы соответствующих подпространств. Эти функции образуют особый класс смещенных базисных систем (СБС), так как они позволяют выполнять спектральный анализ локальных возмущений сигнала, формируя его частотно - временной спектр. Рассмотрим некоторые основные соотношения КМА при $b_{0} \neq 1$, относящиеся к синтезу вейвлет - подобных базисов и вычислению коэффициентов масштабирующего уравнения и их свойствам [10-12].

Пусть $\left\{V_{j}, j=Z\right\}$ образуют последовательность подпространств цепочки КМА. В соответствии с определением КМА найдется такая функция $\varphi \in V_{0}$, которую называют масштабирующей функцией (scaling function), что множество ее сдвигов $\quad \varphi_{0, k}(t)=\varphi\left(t-b_{0} k\right)$ образует ортонормированный базис подпространства $V_{0}$. Более того, оказывается, что для любого $V_{j}$ существует базис

$$
\left\{\varphi_{j, k}(t)=2^{j / 2} \varphi\left(\left(t-2^{-j} b_{0} k\right) / 2^{-j}\right)\right\} .
$$

КМА накладывает на сами функции $\varphi(t)$ определенные и достаточно жесткие требования: поскольку $\varphi \in V_{0}$ и $V_{0} \subset V_{l}$, то $\varphi(t)$ является линейной комбинацией функций $\varphi_{1, n}(t)$

$$
\varphi(t)=\sum_{n} h(n) \sqrt{2} \varphi\left(2 t-b_{0} n\right)
$$

Это выражение называется масштабирующим уравнением (scaling equation, two-scale equation, refinement equation). Коэффициенты $h(n)$ называются масштабирующими. Они использу- ются в качестве импульсных откликов в блоках цифровых фильтров, реализующих быстрые вычислительные алгоритмы дискретных вейвлетных преобразований (discrete wavelet transform - DWT) -ДВП. В образах Фурье уравнение (1) имеет вид:

$$
\begin{aligned}
& \varphi(\omega)=\frac{1}{\sqrt{2}} \sum_{n} h(n) e^{-i n b_{0} \omega / 2} \varphi\left(\frac{\omega}{2}\right)= \\
& =m_{b_{0}}\left(\frac{\omega}{2}\right) \varphi\left(\frac{\omega}{2}\right)
\end{aligned}
$$

где

$$
m_{b_{0}}(\omega)=\frac{1}{\sqrt{2}} \sum_{n} h(n) e^{-i n b_{0} \omega}=\frac{1}{\sqrt{2}} h(\omega)
$$

$2 \pi / b_{0}$-периодическая функция $m_{b_{0}}(\omega)$ полностью определяет масштабирующую функцию. Базисом для подпространства вейвлетов $W_{j}$ $\left(V_{j+1}=V_{j} \oplus W_{j}\right) \quad$ являются функции $\left\{\psi_{j, k}(t)=2^{j / 2} \psi\left(\left(t-2^{-j} b_{0} k\right) / 2^{-j}\right)\right\}$,

образованные сдвигом и масштабированием одной вейвлетной функции $\psi(t)$.

Так как $W_{0} \in V_{l}$, то $\psi(t)$ может быть представлена в виде взвешенной суммы функций $\varphi_{1, n}(t)$ :

$$
\begin{aligned}
& \psi(t)=\sum_{n} g(n) \sqrt{2} \varphi\left(2 t-b_{0} n\right) \\
& g(n)=(-1)^{1-n} h(1-n) .
\end{aligned}
$$

Разрешающую способность и чувствительность предлагаемых подходов оценивали с помощью набора из четырех пиков гауссовой формы $a_{s} \exp \left\{-\left(t-t_{s}\right)^{2} / 2 \mu_{s}\right\}$. Модель определена на сетке из $\mathrm{N}=2^{10}$ точек $(\mathrm{t}=[1: 1024])$ при $\mu_{s}=20$; интенсивности $a_{s}=\left[\begin{array}{llll}0.25 & 0.7 & 1 & 0.35\end{array}\right]$; положении пиков $\mathrm{t}_{\mathrm{s}}=\left[\begin{array}{l}200 \\ 0\end{array}\right.$ $\left.200+6.6 \mu_{s} 580580+10.2\right]$. Из анализа качества восстановления входного сигнала $x(t)$ по наблюдаемому сигналу $y(t)$ (таблица 1) в зависимости от уровня шума (отношение сигнал/шум по входу при моделировании изменялось от 15 до 150) сделаны следующие выводы (все ошибки восстановления вычислялись по формуле (6) и усреднялись по 50 реализациям шума):

$$
\varepsilon=\operatorname{RMSE}\left(x_{0}-\breve{x}\right)=\left\{\frac{\int\left(x_{0}(t)-\breve{x}(t)\right)^{2} d t}{\int\left|x_{0}(t)\right|^{2} d t}\right\}^{\frac{1}{2}}
$$

- систематическая ошибка (без шума) не превышает $1 \%$;

- увеличение отношения сигнал/шум 8кратное для гауссовой модели;

- несовпадение формы сигнала и аппаратной функции приводит к увеличению СКО восстановления примерно на $20 \%$ и к ухудшению подавлению шума на $25 \%$. 


\begin{tabular}{llllll} 
& ISRA (India) $=\mathbf{3 . 1 1 7}$ & SIS (USA) & $=\mathbf{0 . 9 1 2}$ & ICV (Poland) & $=\mathbf{6 . 6 3 0}$ \\
Impact Factor: & ISI (Dubai, UAE) $=\mathbf{0 . 8 2 9}$ & PUHL (Russia) $=\mathbf{0 . 1 5 6}$ & PIF (India) & $=\mathbf{1 . 9 4 0}$ \\
& GIF (Australia) $=\mathbf{0 . 5 6 4}$ & ESJI (KZ) & $=\mathbf{8 . 7 1 6}$ & IBI (India) & $=\mathbf{4 . 2 6 0}$ \\
& JIF & $\mathbf{1 . 5 0 0}$ & SJIF (Morocco) $=\mathbf{5 . 6 6 7}$ & OAJI (USA) & $\mathbf{0 . 3 5 0}$ \\
\hline
\end{tabular}

Таблица 1. КО восстановления и увеличение отношения сигнал/шум

\begin{tabular}{|l|l|l|l|l|l|}
\hline Отношение сигнал/шум на входе & Без шума & 150 & 60 & 30 & 15 \\
\hline Увеличение отношения сигнал/шум & - & 4 & 7 & 8 & 9 \\
\hline $\begin{array}{l}\text { Относительная ошибка } \\
\text { восстановления }\end{array}$ & 0.0069 & 0.0087 & 0.0162 & 0.027 & 0.035 \\
\hline
\end{tabular}

Из анализа качества оценки параметров пиков (положения и амплитуды) при различных отношениях сигнал/шум (рисунок 2) до обработки и после нее сделаны следующие выводы:

- если до обработки некоторые пики не обнаруживаются (из-за шума), то после обработки они обнаруживаются все со случайной ошибкой по интенсивности не более $2 \%$ и по положению одна-две дискреты при отношении сигнал/шум 15;

- оценка положения пиков смещенная. Величина смещения не более трех дискрет и зависит от положения пика по отношению к большему соседнему пику;

- оценка интенсивности пиков также смещенная. Величина смещения составляет до $12 \%$ при отношении сигнал/шум 7. При увеличении отношения сигнал/шум в 20 раз, величина смещения меняет знак на отрицательный и уменьшается до 80 раз.

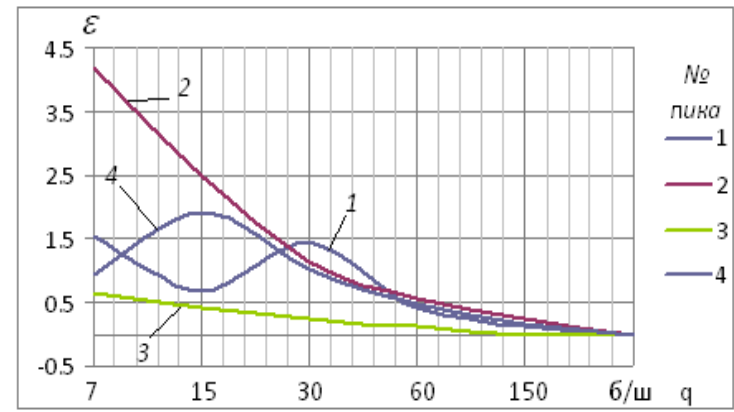

a) СКО положения пиков;

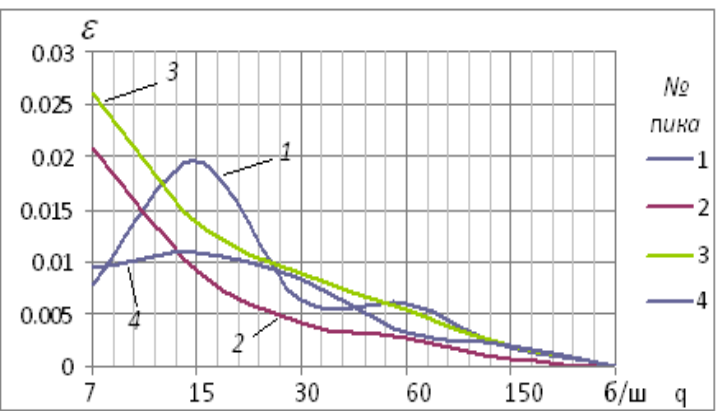

б) СКО интенсивности пиков

Рисунок 2. СКО положения и интенсивности пиков

\section{References:}

1. Tulenbayev, M. S., \& Tulenbayev, Z. S. (2000). Spektralnii podhod k obrabotke informacii. - $V$ zhurnale Nauka i obrazovanie IUzhnogo Kazahstana, №22, pp.262-264.

2. Tulenbayev, M. S., Tulenbayev, K. S., \& Seraliev, T. A. (2000). Modelirovanie, vosstanovlenie i obrabotka signalov analiticheskih priborov v usloviyah pomeh. $V$ zhurnale Mehanika i modelirovanie processov $i$ tehnologii, №1, pp.47-49.

3. Rusinov, L. A. (1984). Avtomatizaciya analiticheskih sistem opredeleniya sostava $i$ kachestva veshestv. (p.160). L.: Himiya.

4. Rusinov, L. A., \& Tulenbayev, M. S. (1984). Kompleksnii algoritm pervichnoi obrabotki signalov pervogo klassa. - V kn. Spektralnii podhod $\mathrm{k}$ pervichnoi obrabotke signalov analiticheskih priborov/Pod red. A.L.Gurevicha. (Eds.). (p.160). L.: Izd-vo Leningr. Un-ta.

5. Tulenbayev, M. S. (2010). Spektralnie algoritmi obrabotki signalov himiko-analiticheskih kompleksov $\mathrm{v}$ geoinformacionnih sistemah ekomonitoringa. Vestnik NAN RK, Almati, 2010. №1, pp.11-17.

6. Novikov, L. V. (2006). Modificirovannie veivleti $\mathrm{v}$ obrabotke dannih anali-ticheskih priborov. Nauchnoe priborostroenie, T.16, №1, pp.3-14. 


\begin{tabular}{llllll} 
& ISRA (India) $=\mathbf{3 . 1 1 7}$ & SIS (USA) $=\mathbf{0 . 9 1 2}$ & ICV (Poland) & $\mathbf{= 6 . 6 3 0}$ \\
Impact Factor: & ISI (Dubai, UAE) $=\mathbf{0 . 8 2 9}$ & PUHЦ (Russia) $=\mathbf{0 . 1 5 6}$ & PIF (India) & $=\mathbf{1 . 9 4 0}$ \\
& GIF (Australia) $=\mathbf{0 . 5 6 4}$ & ESJI (KZ) & $\mathbf{8 . 7 1 6}$ & IBI (India) & $=\mathbf{4 . 2 6 0}$ \\
& JIF & $\mathbf{1 . 5 0 0}$ & SJIF (Morocco) $=\mathbf{5 . 6 6 7}$ & OAJI (USA) & $\mathbf{0 . 3 5 0}$ \\
\hline
\end{tabular}

7. Trahtman, A. M. (1972). Vvedenie $v$ obobshennuiu spektralnuiu teoriiu signalov. (p.351). Moscow: Sov. Radio.

8. Pugachev, V. S. (1962). Teoriya sluchainih funkcii. (p.884). Moscow: Fizmatgiz.

9. Perov, V. P. (1982). Prikladnaya spektralnaya teoriya ocenivaniya. (p.432). Moscow: Nauka.

10. Tulenbayev, M. S. (2009). Sintez adaptivnih bazisov spektralnogo predstav-leniya signalov himiko-analiticheskih kompleksov - zh/l Vestnik TarGU im. M.H.Dulati "Prirodopolzovanie $i$ problemi antroposferi", Тараз 2009, №3, pp.189-196.
11. Novikov, L. V. (2007). Obrabotka signalov na osnove ortonormirovannih kvaziveivletov. Priborostroenie: Izv. Vuzov, T.50, №1, pp.3-10.

12. Tulenbayev, M. S., Beglerova, S. T., Zhuleva, L. V., \& Makoveckaya, A. A. (2014). Adaptive wavelet technolo-gies of chemical and analytical information systems of ecomonitoring International Scientific Practical Conference " European Scientific Achievements", 30.10.2014, Brigton, UK: International scientific journal «Theoretical \& Applied Science», №10 (18), pp. 59-67. 